\title{
A national survey of valganciclovir dosing strategies in pediatric organ transplant recipients
}

\author{
Suhail Shaikh ${ }^{1}$ (D) | Natalia Jasiak-Panek ${ }^{2}$ | Jeong M. Park ${ }^{3}$
}

${ }^{1}$ Department of Pharmacy, MedStar Georgetown University Hospital, Washington, District of Columbia

${ }^{2}$ Department of Pharmacy, Ann \& Robert H. Lurie Children's Hospital of Chicago,

Chicago, Illinois

${ }^{3}$ Department of Clinical Pharmacy, College of Pharmacy, University of Michigan, Ann Arbor, Michigan

Correspondence

Suhail Shaikh, Department of Pharmacy, MedStar Georgetown University Hospital, Washington, DC.

Email: suhail.a.shaikh@gunet.georgetown. edu.

\begin{abstract}
Purpose: Data remain limited on the most appropriate valganciclovir (VGCV) dosing strategy for cytomegalovirus (CMV) prophylaxis and treatment in pediatric organ transplant recipients. Therefore, the objective of this study was to describe methods used to calculate VGCV dosing among pediatric transplant centers.

Methods: A survey of pharmacists was conducted to assess VGCV dosing strategies for $\mathrm{CMV}$ prophylaxis and treatment among pediatric organ transplant centers in the U.S.

Findings: Of 54 centers that perform pediatric organ transplants, $22(40.7 \%)$ centers responded to the survey. Fourteen centers (53.8\%) utilize the Food and Drug Administration (FDA) recommended VGCV dosing strategy of $7 \times$ body surface area $(B S A) \times$ creatinine clearance $(\mathrm{CrCl})$ for $\mathrm{CMV}$ prophylaxis. Other dosing strategies reported included weight based and $520 \mathrm{mg} / \mathrm{m}^{2} \times$ BSA per day. Dosing strategies of VGCV for the treatment of CMV also differed across centers, with a majority (43.5\%) using $7 \times \mathrm{BSA} \times \mathrm{CrCl}$ twice daily.

Conclusion: Less than two-thirds of centers utilize the FDA-approved daily dosing regimen with various methods of $\mathrm{CrCl}$ calculation and serum creatinine assay measurements used. More retrospective and prospective studies with clinical outcomes associated with VCGV dosing strategies are warranted to determine the most appropriate prophylaxis and treatment strategies for CMV.
\end{abstract}

\section{KEYWORDS}

antibiotic: antiviral-ganciclovir/valganciclovir, complication: infectious, infection and infectious agents, viral, infection and infectious agents, viral: cytomegalovirus

\section{1 | INTRODUCTION}

Valganciclovir (VGCV) is extensively used for cytomegalovirus (CMV) infection prophylaxis and treatment following solid organ transplantation (SOT) and was approved by the Food and Drug Administration (FDA) for the prevention of CMV infection in adult and pediatric heart ( 1 month to 16 years of age) and kidney ( 4 months to 16 years of age) transplant recipients. ${ }^{1}$

In 2009, the FDA approved the Pescovitz dosing algorithm of $7 \times$ body surface area $(\mathrm{BSA}) \times \mathrm{CrCl}$ (derived from a Modified Schwartz formula) for VGCV prophylaxis in pediatric patients. ${ }^{2}$ The FDA approved duration for prophylaxis is 100 days in kidney transplant recipients and 200 days in heart recipients. Importantly, the package insert states that the $k$ values used in the Schwartz equation are based on the Jaffe method of measuring serum creatinine $(\mathrm{Scr})$, and may require correction when enzymatic methods are used. The final dose of VGCV calculated may be significantly impacted if the $k$ value is not corrected when a Jaffe method is not used to measure Scr. Several other dosing algorithms have been suggested based on weight and BSA; however, these studies are not correlated to clinical outcomes. ${ }^{2-4}$.

The FDA issued a safety alert warning in 2010 that the FDA approved dosing algorithm could result in overdosing in pediatric 
patients with a low body weight, low BSA, and normal creatinine values. ${ }^{5}$ In this safety alert, the FDA recommended using a maximum $\mathrm{CrCl}$ of $150 \mathrm{~mL} / \mathrm{min} / 1.73 \mathrm{~m}^{2}$ if the calculated Schwartz creatinine clearance $(\mathrm{CrCl})$ exceeded $150 \mathrm{~mL} / \mathrm{min} / 1.73 \mathrm{~m}^{2}$, and using a maximum daily dose of $900 \mathrm{mg}$ if the calculated dose exceeded $900 \mathrm{mg}$. The clinical trials performed by the manufacturer leading to the current FDA approved VGCV dosing algorithm for CMV prophylaxis did not specifically analyze the subpopulation of patients with low body weight, low BSA, and normal creatinine values. ${ }^{4,6}$ They also did not comment on the assay used to measure $\mathrm{Scr}$, which may have significantly impacted final dosing. Due to these limitations, and suggestion of other dosing schemes, there is no consensus regarding the most appropriate dosing algorithm of VGCV for CMV prophylaxis and treatment in pediatric SOT recipients. Therefore, the objective of this study was to describe the methods used to calculate VGCV dosing among pediatric transplant centers for both CMV prophylaxis and treatment.

\section{2 | MATERIALS AND METHODS}

We sent a survey via the Internet in November 2017 to pharmacists working in 54 transplant centers identified by the Scientific Registry of Transplant Recipients across the United States. We developed this survey to assess center practices surrounding prophylaxis and treatment of CMV in pediatric transplant recipients. Specifically, the following objectives for this survey were established:

1. To identify the most common method used to calculate VGCV dosing for CMV prophylaxis and treatment.

2. To determine the most common method used to calculate $\mathrm{CrCl}$ for VGCV dosing.

3. To identify the most common laboratory method used to measure Scr.

4. To assess the duration of VGCV prophylaxis used.

The survey was generated using Qualtrics software (Qualtrics, Provo, UT), which utilizes a web-based interface. The survey included 10 questions and was designed with branch logic questioning based so that respondents were not required to answer questions that did not pertain to their practice based on previous answers. The MedStar Georgetown University Hospital IRB approved the survey.

\section{3 | RESULTS}

There were 24 respondents, with two centers having multiple responses (two responses each); both responses were merged into one consensus response for each of the two centers if responses were identical. Responses were received for kidney $(n=20)$, liver ( $n=15)$, heart $(n=9)$, small bowel $(n=4)$, and lung $(n=1)$ transplants.
Responses were not delineated by organ, rather cumulative responses are reported. Responding centers varied from low to high volume centers, as well as geographic location.

\subsection{CMV prophylaxis dosing strategies}

There were 26 responses received from the 22 centers regarding the method used to calculate VGCV dosing for CMV prophylaxis. A majority of responders (53.8\%) utilize the FDA approved daily dosing equation of $7 \times \mathrm{BSA} \times \mathrm{CrCl}$. Other responses included using $520 \mathrm{mg} /$ $\mathrm{m}^{2}\left(900 \mathrm{mg} / 1.73 \mathrm{~m}^{2}\right) \times$ BSA per day and weight-based dosing ranging from 10 to $25 \mathrm{mg} / \mathrm{kg} / \mathrm{d}$ (Figure 1). Three centers reported using different equations depending on the organ transplanted.

Only 6 of the responders utilizing the FDA approved dosing scheme used a maximum $\mathrm{CrCl}$ of $150 \mathrm{~mL} / \mathrm{min} / 1.73 \mathrm{~m}^{2}$, whereas seven responders used a maximum $\mathrm{CrCl}$ of $120 \mathrm{~mL} / \mathrm{min} / 1.73 \mathrm{~m}^{2}$. One center reported not using a maximum $\mathrm{CrCl}$. Of the 14 responders using the FDA approved dosing equation, 8 (57.1\%) reported using the modified Schwartz equation $(\mathrm{CrCl}=\mathrm{k} \times$ height/Crserum), while 5 (35.7\%) use the bedside Schwartz equation (GFR $=0.413 \times$ height/ Crserum). One responder reported using the modified Schwartz equation for heart transplant recipients, but the bedside Schwartz for kidney transplant recipients. Eighteen centers reported using a maximum VGCV dose of $900 \mathrm{mg}$, whereas three centers reported a maximum dose of $450 \mathrm{mg}$ daily.

\subsection{Serum creatinine assay}

Overall, a majority of responders (66.7\%) were unsure of the assay their center uses to measure Scr. Four centers use a method traceable to an isotope dilution mass spectrometry (IDMS), and three centers use a corrected or modified Jaffe by calibration traceable to IDMS. Of the centers using the FDA approved dosing equation, two $(14.3 \%)$ responders reported using IDMS, two (14.3\%) reported using corrected/modified Jaffee by calibration to IDMS, and the remaining responders (71.4\%) were unsure of the method used to measure Scr.

\section{3 | Duration of valganciclovir prophylaxis}

The duration of prophylaxis reported varied significantly based on the donor and recipient CMV serostatus as well as the type of organ transplanted. We gathered information for each possible CMV serostatus (Table 1). A majority of responders (53.1\%) used VGCV prophylaxis for 180 days post-transplantation in high-risk serostatus (CMV donor positive/recipient negative) patients.

\section{4 | CMV treatment dosing strategies}

Although VGCV is not FDA approved for the treatment of CMV in pediatric patients, it is extensively used; therefore, we also surveyed pharmacists to determine the most common VGCV dosing strategy used for treatment of CMV. Nine responders (39.1\%) reported using 
FIGURE 1 Methods used to calculate valganciclovir dose for CMV prophylaxis $(\mathrm{N}=26)$

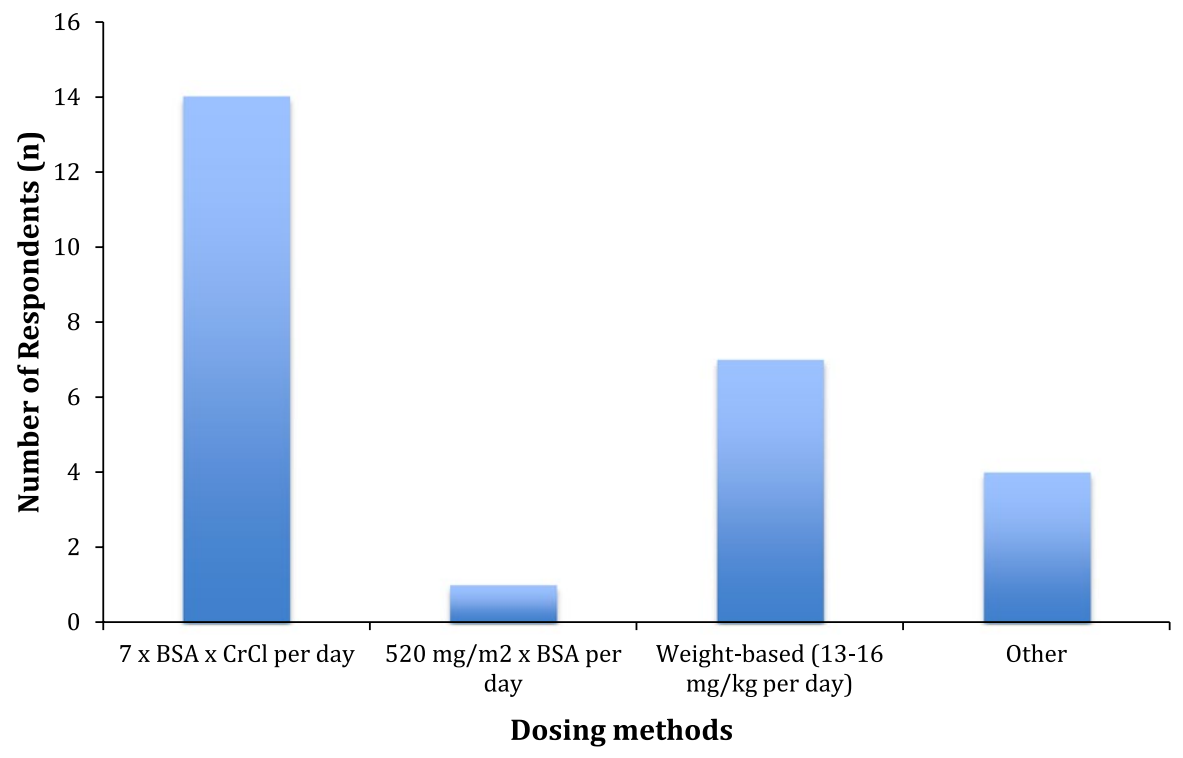

the $7 \times \mathrm{BSA} \times \mathrm{CrCl}$ twice daily method to calculate VGCV treatment dosing, 6 (26.1\%) use IV ganciclovir and not VGCV for treatment, 4 (17.4\%) use weight-based dosing (13-16 mg/kg per dose), and 3 (13.0\%) use age-specific dosing.

\section{4 | DISCUSSION}

We report the results of the first comprehensive survey addressing the center-specific practices and policies regarding VGCV prophylaxis for $\mathrm{CMV}$ in pediatric transplant recipients. The FDA approved dosing equation $(7 \times \mathrm{BSA} \times \mathrm{CrCl})$ was utilized in less than $60 \%$ of survey respondents; moreover, significant variability was observed in methods of $\mathrm{CrCl}$ calculation and maximum $\mathrm{CrCl}$ value utilized among them. Only six responding centers utilize the FDA approved dosing equation and recommended maximum $\mathrm{CrCl}$ when dosing VGCV. However, most centers did report using a maximum daily dose of $900 \mathrm{mg}$ in high-risk CMV serostatus patients. Our data indicate that there is significant center variability in methods used to calculate VGCV dosing. We also found significant variability within centers based on type of organ transplant.

Several dosing algorithms for VGCV have been suggested based on body surface area (BSA), weight, or incorporating both BSA and $\mathrm{CrCl}$; however, none of these studies directly correlate dosing method to clinical outcomes. ${ }^{2-4}$ Furthermore, dosing VGCV in the pediatric patient population can be challenging due to multiple factors such as diet, metabolic activity, and renal function effecting principle pharmacokinetic properties. ${ }^{7}$ These challenges may lead to overdosing VGCV that may lead to severe neutropenia, anemia, or thrombocytopenia. Additionally, under-dosing VGCV increases risk of CMV disease and viral resistance. The results of this survey demonstrate that many transplant centers are modifying VGCV dosing, possibly in attempt to address the concerns in patients with low body weight, low BSA, and normal creatinine values. This includes using weight based dosing of $13-16 \mathrm{mg} / \mathrm{kg}$ per day or $520 \mathrm{mg} / \mathrm{m}^{2}$ per day. Adult VGCV dosing is well established, as strategies are based on clinical outcomes data as well as AUC values shown to prevent and treat CMV disease. ${ }^{1,2,8}$ However, the pharmacokinetic and outcomes data in pediatric patients is limited, with the few available studies in pediatric patients varying in age range of patients, organ transplanted, indication for VGCV, and dosing regimen. ${ }^{9}$

Several factors can explain this lack of standardization including no unanimity of optimal target ganciclovir $\mathrm{AUC}_{0-24}$ values. ${ }^{10-12}$ Low dosing regimens are supported by findings of clinical efficacy, possible immunologic protection, and reduced toxicity, whereas higher exposure is supported by better suppression of subclinical disease and seroconversion. ${ }^{9}$ As mentioned previously, VGCV pharmacokinetic and outcomes data in pediatric patients are limited. However, what is known is the significant inter- and intrapatient variability of ganciclovir concentrations in the population., ${ }^{9,13}$

Multiple variables are included in the FDA approved dosing strategy for VGCV including patient height, weight, Scr, coefficients according to sex and age if using the modified Schwartz equation, and importantly the method used to measure Scr, an important factor that may often be overlooked. In 2002, the Clinical Practice Guidelines of the National Kidney Foundation Kidney Disease Outcomes Quality Initiative (NKF KDOQI) recommended the use of the traditional, now modified, Schwartz equation to estimate GFR in children. ${ }^{14}$ Now that many major global manufacturers in the USA and other countries have calibration traceable to an IDMS to measure Scr via enzymatic methods, using the modified Schwartz equation overestimates GFR by $20 \%-40 \% .{ }^{14}$ Therefore, in 2009 , the formula was updated to the bedside Schwartz equation, which was devised for use with creatinine methods with calibration traceable to IDMS. This is now considered by NKF KDOQI to be the most appropriate method to estimate GFR in children. As mentioned previously, the VGCV package insert recommends using the modified Schwartz equation to calculate $\mathrm{CrCl}$ for the dosing algorithm. The package insert also states that the $\mathrm{k}$ values used in the modified Schwartz equation are based on the Jaffe method of measuring Scr and may require correction when 
TAB LE 1 Pediatric VGCV survey results

\begin{tabular}{|c|c|}
\hline Number of responding centers & 22 \\
\hline \multicolumn{2}{|l|}{ Organ type } \\
\hline Kidney & 20 \\
\hline Liver & 15 \\
\hline Heart & 9 \\
\hline Small bowel & 4 \\
\hline Lung & 1 \\
\hline \multicolumn{2}{|c|}{ Method used to calculate VGCV dose for CMV prophylaxis $(n=26)$} \\
\hline $7 \times \mathrm{BSA} \times \mathrm{CrCl}$ & $14(53.8 \%)$ \\
\hline $520 \mathrm{mg} / \mathrm{m}^{2} \times$ BSA per day & $1(3.8 \%)$ \\
\hline Weight based such as $13-16 \mathrm{mg} / \mathrm{kg}$ per day & $7(26.9 \%)$ \\
\hline Other & 4 (15.4\%) \\
\hline \multicolumn{2}{|l|}{ Method used to calculate $\mathrm{CrCl}(\mathrm{n}=22)$} \\
\hline Modified Schwartz $(\mathrm{CrCl}=\mathrm{k} \times$ height $/$ Crserum $)$ & $11(50.0 \%)$ \\
\hline Bedside Schwartz (GFR $=0.413 \times$ height $/$ Crserum) & $10(45.5 \%)$ \\
\hline Other & $1(4.5 \%)$ \\
\hline \multicolumn{2}{|l|}{ Assay used to measure $\operatorname{Scr}(n=21)$} \\
\hline Unsure & $14(66.7 \%)$ \\
\hline IDMS & $4(19.0 \%)$ \\
\hline $\begin{array}{l}\text { Corrected/modified Jaffe by calibration traceable } \\
\text { to IDMS }\end{array}$ & $3(14.3 \%)$ \\
\hline Original Jaffe & 0 \\
\hline \multicolumn{2}{|l|}{ Maximum daily dose of VGCV for CMV prophylaxis $(n=21)$} \\
\hline $900 \mathrm{mg}$ & $18(85.7 \%)$ \\
\hline Other & 3 (14.3\%) \\
\hline No maximum dose & 0 \\
\hline
\end{tabular}

VGCV, valganciclovir; CMV, cytomegalovirus; BSA, body surface area; $\mathrm{CrCl}$, creatinine clearance; GFR, glomerular filtration rate; Scr, serum creatinine; IDMS, isotope dilution mass spectrometry.

Data are represented as $\mathrm{n}$, and $\mathrm{n}(\%)$.

enzymatic methods are used; however, do not provide information on how to correct. The clinical trials performed by the manufacturer leading to the current FDA approved VGCV dosing algorithm in children (Pescovitz formula) for CMV prophylaxis used the modified Schwartz equation to calculate $\mathrm{CrCl}$; however, they do not provide information on whether Scr was measured based on a Jaffe method or enzymatic method, which is a significant limitation. ${ }^{4,6}$

Asberg and colleagues evaluated VGCV-dosing algorithms in pediatric solid organ transplant recipients, including the Pescovitz formula $($ VGCV dose $=7 \times \mathrm{BSA} \times \mathrm{CrCl}) .{ }^{15}$ Using data from 104 pediatric transplant recipients (including kidney, liver, and heart transplant), the researchers performed Monte Carlo simulations to investigate the probability of achieving a ganciclovir AUC of $40-60 \mathrm{mg} \times \mathrm{h} / \mathrm{L}$ with various dosing algorithms. The authors concluded that current dosing algorithms are only accurate in about $33 \%$ of patients. The Pescovitz algorithm tends to overdose young children and under dose older children. The authors concluded that the Cockcroft-Gault formula outperformed the Schwartz equation, as it was a better descriptor for ganciclovir behavior in the pediatric population. Using a
TABLE 2 Duration of CMV prophylaxis according to CMV serostatus

\begin{tabular}{|c|c|}
\hline CMV serostatus & $n(\%)$ \\
\hline \multicolumn{2}{|l|}{$C M V D+/ R-(N=32)$} \\
\hline 12 months & $5(15.6)$ \\
\hline 6-12 months & $1(3.1)$ \\
\hline 6 months & $17(53.1)$ \\
\hline 4 months & $1(3.1)$ \\
\hline 3 months & $8(25.0)$ \\
\hline Pre-emptive approach & 0 \\
\hline No prophylaxis & 0 \\
\hline \multicolumn{2}{|l|}{$C M V D+/ R+(N=30)$} \\
\hline 12 months & $4(13.3 \%)$ \\
\hline 6-12 months & 0 \\
\hline 6 months & 9 (30.0\%) \\
\hline 4 months & $1(3.3 \%)$ \\
\hline 3 months & 15 (50.0\%) \\
\hline Pre-emptive approach & 1 (3.3\%) \\
\hline No prophylaxis & 0 \\
\hline \multicolumn{2}{|l|}{ CMV D-/R+ $(N=29)$} \\
\hline 12 months & $4(13.8)$ \\
\hline 6-12 months & 0 \\
\hline 6 months & $8(27.6)$ \\
\hline 4 months & $1(3.4)$ \\
\hline 3 months & $15(51.7)$ \\
\hline Pre-emptive approach & $1(3.4)$ \\
\hline No prophylaxis & 0 \\
\hline \multicolumn{2}{|l|}{ CMV D-/R- $(N=19)$} \\
\hline 12 months & $1(5.3)$ \\
\hline 6-12 months & 0 \\
\hline 6 months & $4(21.1)$ \\
\hline 4 months & 0 \\
\hline 3 months & $11(57.9)$ \\
\hline Pre-emptive approach & $1(5.3)$ \\
\hline No prophylaxis & $2(10.5)$ \\
\hline
\end{tabular}

CMV, cytomegalovirus.

Data are represented as $\mathrm{n}(\%)$.

nonparametric model, the authors suggest the following equation for estimating initial VGCV prophylactic dosing in children:

Dose $(\mathrm{mg})=$ body weight $(\mathrm{kg}) \times(0.07 \times \mathrm{GFR}+\mathrm{k} *)$

${ }^{*} k=5$ for GFR $\leq 30 \mathrm{~mL} / \mathrm{min}, k=10$ for GFR $>30 \mathrm{~mL} / \mathrm{min}$ and weight $>30 \mathrm{~kg}, k=15$ for GFR $>30 \mathrm{~mL} / \mathrm{min}$ and weight $\leq 30 \mathrm{~kg}$.

The authors further recommend ganciclovir therapeutic drug monitoring for subsequent doses and state that without such monitoring only $20 \%$ of patients will achieve a therapeutic AUC.

Asberg's study is limited by their simulation design while our study's limitations are related to sample size and the survey-based methodology. Only $40.7 \%$ of transplant centers performing pediatric 
organ transplants identified within UNOS responded to the survey, which may underrepresent the most common methodology to calculate VGCV dosing. Surveys are significantly limited by the amount of information that can be gathered from respondents, and many of the centers $(66.7 \%)$ completing our survey were not aware of the type of method was used by their institution to measure Scr. Although these limitations are not ideal, this is the only assessment of center practices regarding VGCV dosing strategies and duration for CMV prophylaxis and treatment of pediatric transplant centers in the USA (Table 1 and 2).

This study highlights VGCV dosing strategies for CMV prophylaxis used across multiple U.S. pediatric transplant centers that responded to the survey and demonstrates the variability in practices. Many factors influence the dose of VGCV in pediatric patients, including the equation used to calculate dose, the equation used to calculate $\mathrm{CrCl}$, the laboratory assay method used to measure $\mathrm{Scr}$, and patient pharmacodynamic characteristics. Subtle differences can significantly impact the final calculated dose of VGCV, which may lead to either over-dosing which may result in significant leukopenia, or underdosing which may lead to inadequate prophylaxis and development of resistant $\mathrm{CMV}$. With so many variables influencing dosing, it is no surprise that there is no consensus across transplant centers. Our survey results support the continued need for more data surrounding VGCV use in pediatric patients to allow for a national consensus on an optimal dosing strategy. Specifically a randomized trial comparing the use of the modified Schwartz equation to the bedside Schwartz equation on incidence of CMV viremia and leukopenia may generate answers on the most efficacious and safest dosing strategy for $\mathrm{CMV}$ prophylaxis.

\section{ACKNOWLEDGEMENTS}

No funding was required to support the production of this manuscript.

\section{DISCLOSURE}

The authors of this manuscript have no conflicts of interest to disclose.

\section{ORCID}

Suhail Shaikh (iD http://orcid.org/0000-0002-2438-0349

\section{REFERENCES}

1. Valcyte ${ }^{\circledR}$ [package insert]. San Francisco, CA: Genentech, Inc; 2017.
2. Pescovitz MD, Ettenger RB, Strife CF, et al. Pharmacokinetics of oral valganciclovir solution and intravenous ganciclovir in pediatric renal and liver transplant recipients. Transpl Infect Dis. 2010;12:195-203.

3. Launay E, Theoret $\mathrm{Y}$, Litalien $\mathrm{C}$, et al. Pharmacokinetic profile of valganciclovir in pediatric transplant recipients. Pediatr Infect Dis J. 2012;31:405-407.

4. Villeneuve D, Brothers A, Harvey E, et al. Valganciclovir dosing using area under the curve calculations in pediatric solid organ transplant recipients. Pediatr Transpl. 2013;17:80-85.

5. FDA Drug Safety Communication: New dosing recommendations to prevent potential Valcyte (valganciclovir) overdose in pediatric transplant patients. 2010. https://www.fda.gov/Drugs/ DrugSafety/ucm225727.htm. Accessed May 25, 2018.

6. Vaudry W, Ettenger R, Jara P, et al. Valganciclovir dosing according to body surface area and renal function in pediatric solid organ transplant recipients. Am J Transplant. 2009;9:636-643.

7. Asberg A, Humar A, Rollag H, et al. VICTOR Study Group. Oral valganciclovir is noninferior to intravenous ganciclovir for the treatment of cytomegalovirus disease in solid organ transplant recipients. Am J Transplant. 2007;7:2106-2113.

8. Wiltshire H, Hirankarn S, Farrell C, et al. Pharmacokinetic profile of ganciclovir after its oral administration and from its prodrug, valganciclovir, in solid organ transplant recipients. Clin Pharmacokin. 2005;44:495-507.

9. Peled O, Berkovitch M, Rom E, et al. Valganciclovir dosing for cytomegalovirus prophylaxis in pediatric solid-organ transplant recipients. Pediatr Infect Dis J. 2017;36:745-750.

10. Arasaratnam RJ. Lower-dose valganciclovr for the prevention of cytomegalovirus after solid organ transplantation: an important tradeoff. Transpl Infect Dis. 2015;17:623-624.

11. Stevens DR, Sawinski D, Blumberg E, et al. Increased risk of breakthrough infection among cytomegalovirus donor-positive/ recipient-negative kidney transplant recipients receiving lowerdose valganciclovir prophylaxis. Transpl Infect Dis. 2015;17:163-173.

12. Vezina HE, Brundage RC, Balfour HH Jr. Population pharmacokinetics of valganciclovir prophylaxis in paedriatric and adult solid organ transplant recipients. Br J Clin Pharmacol. 2014;78:343-352.

13. Vethamuthu J, Feber J, Chretein A, et al. Unexpectedly high interand intrapatient variability of ganciclovir levels in children. Pediat Transplant. 2007;11:301-305.

14. K/DOQI clinical practice guidelines for chronic kidney disease: evaluation, classification, and stratification. Am J Kidney Dis. 2002;39:S1-S266.

15. Asberg A, Bjerre A, Neely M. New algorithm for VGCV dosing in pediatric solid organ transplant recipients. Pediatr transplantation. 2014;18:103-111.

How to cite this article: Shaikh S, Jasiak-Panek N, Park JM. A national survey of valganciclovir dosing strategies in pediatric organ transplant recipients. Clin Transplant. 2018;32:e13369. https://doi.org/10.1111/ctr.13369 